\title{
Low-dose gonadotropin induction of ovulation in anovulatory women: still needed in the age of IVF
}

\author{
Davinia M White ${ }^{1, *}$, Kate Hardy $2, *$, Suzannah Lovelock ${ }^{2}$ and Stephen Franks² \\ ${ }^{1}$ Reproductive Medicine Clinic, Imperial College Healthcare NHS Trust, St Mary's Hospital, London, UK and \\ ${ }^{2}$ Institute of Reproductive \& Developmental Biology, Imperial College London, Hammersmith Hospital, London, UK
}

Correspondence should be addressed to S Franks; Email: s.franks@imperial.ac.uk

*(D M White and K Hardy contributed equally to this work)

This paper is part of an Anniversary Issue celebrating 40 years of in vitro fertilisation. The Guest Editor for this section was Professor Lord Robert Winston.

\begin{abstract}
Low-dose, step-up gonadotropin is the treatment of choice for women with polycystic ovary syndrome (PCOS) who have not conceived after anti-oestrogen treatment and as an effective alternative to pulsatile GnRH in women with hypogonadotropic hypogonadism $(\mathrm{HH})$. There has been, however, no large-scale, comparative study between the two groups using low-dose gonadotropins. Here, we performed a retrospective, comparative analysis, in a single clinic database, of efficacy and safety of induction of ovulation using low-dose gonadotropins in 364 women with PCOS and 80 women with $\mathrm{HH}$. The rate of ovulation was high in both PCOS $(83 \%)$ and $\mathrm{HH}(84 \%)$ but mono-follicular, ovulatory cycles were more prevalent in PCOS than in $\mathrm{HH}(77 \%$ vs $53 \%, P<0.0001)$ and the proportion of cycles that were abandoned was higher in $\mathrm{HH}$ than in PCOS $(25 \%$ vs $15 \%, P<0.0001)$. The median threshold dose of gonadotropin required to induce ovulation was $75 \mathrm{IU} /$ day in PCOS and $113 \mathrm{IU} / \mathrm{day}$ in $\mathrm{HH}(P<0.001)$ and the range of doses was greater in $\mathrm{HH}$ women. Forty-nine percent of women with PCOS and $65 \%$ of those with $\mathrm{HH}$ conceived (more than $90 \%$ within 6 cycles of treatment) and had at least one pregnancy. Multiple pregnancies (all twins) occurred in only $4 \%$ of women with PCOS and $5 \%$ of those with $\mathrm{HH}$. These findings emphasise the efficacy and safety of low-dose gonadotropin treatment for both clomiphene-resistant women with PCOS and those with $\mathrm{HH}$. These results highlight the importance of choosing the more physiological approach of gonadotropin induction of ovulation in both groups as the most appropriate treatment, in preference to IVF.

Reproduction (2018) 156 F1-F10
\end{abstract}

\section{Introduction}

The isolation of human gonadotropins, first from pituitary glands (Gemzell et al. 1958, Crooke et al. 1963, 1967, Gemzell 1966) and subsequently menopausal urine (Crooke et al. 1963, Diczfalusy et al. 1964, Lunenfeld \& Insler 1974), heralded a new era of treatment for anovulatory infertility in the late nineteen-fifties and early sixties. Exogenous gonadotropins have since been used, successfully, for induction of ovulation both in women with hypogonadotropic amenorrhoea and those with polycystic ovary syndrome (PCOS) (Gemzell et al. 1958, Crooke et al. 1963, 1967, Diczfalusy et al. 1964, Gemzell 1966, Brown et al. 1967, Lunenfeld \& Insler 1974). Refinements in gonadotropin preparations have included purification of urinary FSH and, more recently, the introduction of recombinant human gonadotropins that are now in common use (Franks \& Gilling-Smith 1994, Zwart-van Rijkom et al. 2002). The availability of the antioestrogen clomiphene citrate in the $1960 \mathrm{~s}$
(Lunenfeld \& Insler 1974) provided a more easily manageable method of ovulation induction for women with PCOS and is still regarded as first-line treatment in this condition (Balen et al. 2016). However, a significant proportion (10-30\%) of patients with PCOS have few or no ovulatory cycles in response to clomiphene whilst others, albeit a smaller proportion, will ovulate but not conceive (Kousta et al. 1997). In such cases, gonadotropin induction of ovulation remains an important and effective choice.

In women with amenorrhoea due to hypothalamic dysfunction, the introduction of pulsatile gonadotropinreleasing hormone $(\mathrm{GnRH})$ provided a more physiological method of induction of ovulation than gonadotropin therapy, with excellent results with regard to ovulation and pregnancy rates (Homburg et al. 1989, Martin et al. 1990). Furthermore, the high proportion of mono-ovulatory cycles during $\mathrm{GnRH}$ treatment is associated with a high rate of singleton pregnancies. Unfortunately, the availability of $\mathrm{GnRH}$ preparations for 
induction of ovulation has recently been reduced and many centres are now returning to the use of exogenous gonadotropins for women with HA. In addition, a small proportion of women with $\mathrm{HH}$ have primary pituitary deficiency of gonadotropins so that $\mathrm{GnRH}$ therapy is usually ineffective.

A major problem with gonadotropin induction of ovulation has been the high incidence of multiple follicle development with the attendant increased risk of multiple pregnancy and of ovarian hyperstimulation syndrome (OHSS) (Gemzell 1966, Crooke 1970, Schwartz et al. 1980, Balen et al. 1994, Franks \& GillingSmith 1994, Tadokoro et al. 1997). These risks can be limited by the use of a low-dose 'step-up' regimen, which was introduced in the 1980s and which, for the last 15-20 years has largely taken the place of conventional (i.e. high dose) gonadotropin induction of ovulation, particularly in women with PCOS (Kamrava et al. 1982, Polson et al. 1987, Homburg et al. 1995, White et al. 1996, Thessaloniki 2008, Li et al. 2010, Balen etal. 2016). Low-dose gonadotropin regimens are now accepted as best practice in the case of clomiphene-resistant PCOS (Thessaloniki 2008, Balen et al. 2016) and there are now plentiful data to demonstrate its efficacy and relative safety in reducing the rates of multiple pregnancy and OHSS. There have, however, been no studies that have compared the results of low-dose gonadotropin induction of ovulation in large series of women with PCOS with those who have $\mathrm{HH}$. Previous studies, comparing the results of treatment with conventional gonadotropin regimens, have been characterised by high rates of multiple pregnancy (in excess of $20 \%$ ) in both groups (Tadokoro et al. 1997). Here, we present the results of our gonadotropin induction of ovulation programme in a large series of women with PCOS or $\mathrm{HH}$, using a low-dose step-up regimen. We show that both are effective fertility treatments with a low incidence of adverse effects but with important differences between the groups in responsiveness to gonadotropins and the factors that influence the outcome.

\section{Patients and methods}

We used our database of 445 patients with infertility due to chronic anovulation who were treated at a single centre (Reproductive Medicine Clinic, St Mary's Hospital, Imperial College Healthcare NHS Trust, London, UK) using low-dose gonadotropin induction of ovulation between 1991 and 2013. Patient data were recorded electronically, with consent, from treatment records, and anonymised before analysis. Of the 445 women, 364 patients had a diagnosis of PCOS and 80 had hypogonadotropic hypogonadism $(\mathrm{HH})$ due to gonadotropin deficiency of either hypothalamic (73 women) or pituitary origin (7 women). The underlying diagnoses in women with $\mathrm{HH}$ were: 66 with idiopathic $\mathrm{HH}(\mathrm{IHH}), 2$ with established Kallmann syndrome
(KS), 2 with additional growth hormone deficiency, 2 with hyperprolactinemia (in whom dopamine agonist treatment was not tolerated) and 1 with exercise-related functional hypothalamic amenorrhea (FHA). Seven women had primary pituitary deficiency following pituitary surgery (5 subjects), Sheehan syndrome (1) or iron deposition in pituitary following treatment for thalassemia (1). Nine of the women with $\mathrm{IHH}$ had previously received treatment with pulsatile $\mathrm{GnRH}$, which resulted in ovulation in 3 (one pregnancy) but no consistent response in the remaining 6.

Women with PCOS had previously received treatment with clomiphene citrate (CC) and had either failed to ovulate $(73 \%)$ or had not conceived after at least 6 ovulatory cycles. Women with $\mathrm{HH}$ were slightly older than those with PCOS; BMI was similar in the two groups. All PCOS women had oligomenorrhoea, very irregular menses or oestrogen-replete amenorrhoea (with or without hyperandrogenism) and had polycystic ovaries on ultrasound. They therefore met the Rotterdam diagnostic criteria (2004). Tubal patency and partner's semen analysis were assessed for all subjects before starting gonadotropin treatment.

\section{Confounding infertility factors}

Additional infertility factors were found in 31 PCOS patients who had an abnormal or damaged Fallopian tube on hysterosalpingography and a further 18 had been found to have endometriosis at laparoscopy. Criteria for inclusion in the study were at least one patent fallopian tube and sperm density $>15$ million/ $\mathrm{mL}$. Only 1 out of $17 \mathrm{HH}$ patients investigated for confounding factors was found to have abnormal fallopian tubes. Abnormal semen analyses were reported in 58 partners of PCOS patients; 54 had a sperm density of $<20$ million $/ \mathrm{mL}$ and 4 had low sperm motility (motility index $<30$ ). None of the partners of women with $\mathrm{HH}$ had sperm abnormalities.

\section{Low-dose gonadotropin regimen}

In women with PCOS, following a baseline scan, a short course of a progestagen (medroxyprogesterone acetate, $5 \mathrm{mg} /$ day) was given to induce a withdrawal bleed if the endometrium was greater than $8 \mathrm{~mm}$ in thickness. PCOS women received either human menopausal gonadotropin (hMG; Menotropin, Menopur, Ferring), purified urinary FSH (uFSH, Metrodin, Serono, Feltham, UK) or recombinant human FSH ( $\mathrm{rFSH}$, Gonal- $F$, Serono or, from 1997, Puregon, Organon, Cambridge, UK), which was routinely started at a dose of $52.5 \mathrm{IU}$ by daily intramuscular injection following the onset of spontaneous or progestagen-induced menses (White et al. 1996). In women with $\mathrm{HH}$, only hMG (menotropin) was used as this contains both $\mathrm{LH}$ and FSH activity, which are both necessary for induction of ovulation in women with gonadotropin deficiency. 
The response to treatment was primarily monitored by ultrasound scanning, performed initially at three- to four-day intervals and, in the preovulatory phase, on alternate days (or daily, if indicated). The starting dose of hMG or FSH was maintained for up to 14 days in the first cycle and the dose only increased (by increments of 25 , initially or $37.5 \mathrm{IU}$ if still no response), if no follicle $>10 \mathrm{~mm}$ was observed by that stage. If necessary, further increments were made at weekly intervals, to a maximum of $300 \mathrm{IU} /$ day. Once a follicle of $\geq 10 \mathrm{~mm}$ was achieved, the dose of hMG ('threshold' dose) was maintained until the follicle had reached a diameter of at least $18 \mathrm{~mm}$. Ovulation was triggered by a single, intramuscular dose of hCG, 5000IU and hMG was stopped. HCG was given routinely because, although some patients will have a spontaneous LH surge, it is not possible to predict this with any reliability. Treatment was discontinued if there were more than three follicles of $15 \mathrm{~mm}$ (or larger) in diameter, to minimise the risk of hyperstimulation and/or multiple pregnancy. All the $\mathrm{HH}$ women received luteal phase support with further doses of hCG 2500IU, 3 days and 6 days after the hCG ovulation trigger. Serum progesterone was measured five to eight days after the hCG trigger. If further cycles of treatment were necessary, hMG was reintroduced at a dose below the previous threshold (and no more than $75 \mathrm{IU} /$ day) and first increment in dose was usually made after seven, rather than 14 days. In women who developed multiple follicles, either a smaller starting dose (i.e. 25 or $37.5 \mathrm{IU}$ ) was given or the dose was increased by only $25 \mathrm{IU}$.

\section{Database}

Records from the clinic database were incorporated into Macintosh FileMaker Pro5 and anonymised data were analysed using FileMaker Pro 11 Database. Statistical analysis was performed using Prism 6 for Mac OS X, version 6.0a (www.graphpad.com). D'Agostino \& Pearson omnibus normality test was used to assign normality. Data that were normally distributed were compared using ANOVA or Student's $t$ test and nonparametric Mann-Whitney or Kruskal-Wallis tests were used to assign significance in comparing data that were not normally distributed. Since most of the indices used were not normally distributed, data are presented as medians with either interquartile ranges or 95\% confidence intervals. Correlation coefficients were calculated using the Pearson correlation test. A $P$ value $<0.05$ was considered significant.

\section{Ethics}

These data were accumulated as part of our induction of ovulation service and, therefore, no specific ethical approval was applicable. However, all data were anonymised when setting up the FileMaker Pro database.
Table 1 Clinical and endocrine characteristics of women with $\mathrm{PCOS}$ and $\mathrm{HH}$ who were treated with gonadotropins.

\begin{tabular}{lccc}
\hline & PCOS $(n=365)$ & HH $(n=80)$ & $\boldsymbol{P}^{*}$ \\
\hline Age & $31(27-34)$ & $33(31-35)$ & $<0.0001$ \\
BMI & $24(22-26)$ & $24(21-26)$ & ns \\
LH (IU/L) & $8.6(5-14)$ & $1.4(0.9-3.0)$ & $<0.0001$ \\
FSH (IU/L) & $5.9(5.0-7.1)$ & $5.0(2.4-7.5)$ & 0.0057 \\
Testosterone (nmol/L) & $2.3(1.8-3.0)$ & $1.2(0.7-1.7)$ & $<0.0001$ \\
Prolactin (U/L) & $237(164-350)$ & $129(102-170)$ & $<0.0001$ \\
TSH (mU/L) & $1.5(1.1-2.3)$ & $1.7(1.0-2.6)$ & ns \\
\hline
\end{tabular}

Data are presented as median (IQR); *Mann-Whitney.

\section{Results}

Women with PCOS were slightly younger than those with $\mathrm{HH}$ but $\mathrm{BMI}$ was similar (Table 1). BMI ranged from 17 to $46 \mathrm{~kg} / \mathrm{m}^{2}$ in women with PCOS and $19-29 \mathrm{~kg} / \mathrm{m}^{2}$ in those with $\mathrm{HH}$. Both baseline (pre-treatment) $\mathrm{LH}$ and FSH were, as expected, significantly lower in women with $\mathrm{HH}$ than in those with PCOS, as were serum testosterone and prolactin (Table 1; all data points are shown in Fig. 1). TSH concentrations were similar in the two groups (Table 1).

All women included in the analysis had at least one completed cycle of gonadotropin treatment and $60 \%$ of women in each group received at least three cycles of treatment. Thirty percent in each group went on to have 6 cycles of treatment, with fewer than $10 \%$ requiring eight or more cycles (Fig. 2) (the aim was for each patient to have up to six ovulatory cycles). The median threshold
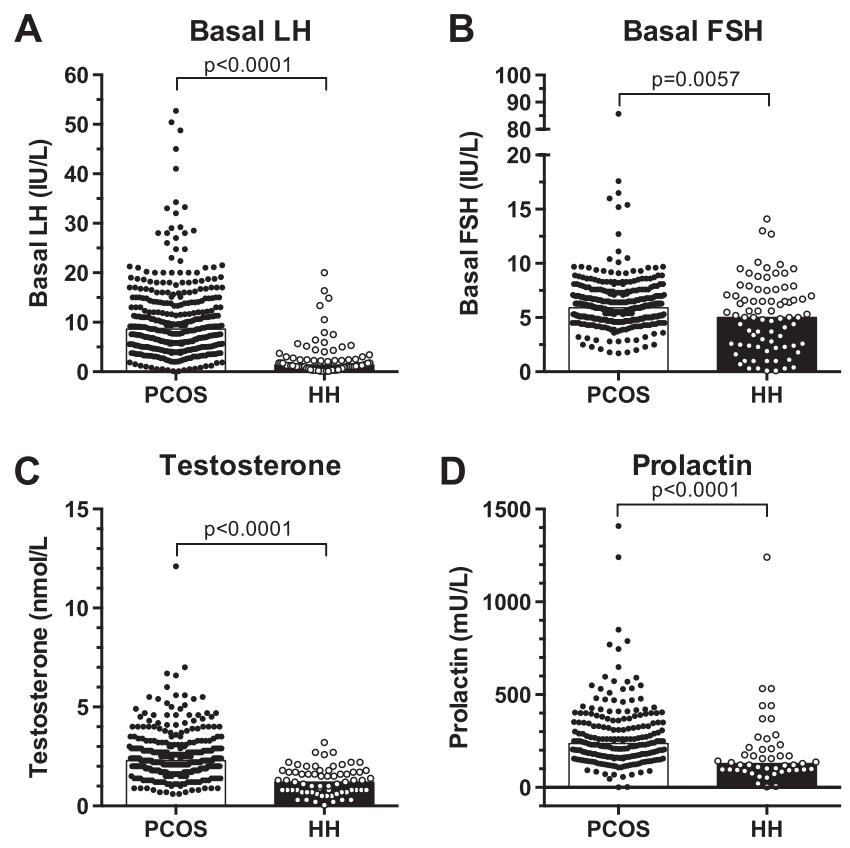

Figure 1 Basal (pre-treatment) endocrine data in women with PCOS or $\mathrm{HH}$ who received gonadotropin treatment (A: $\mathrm{LH}, \mathrm{B}: \mathrm{FSH}, \mathrm{C}$ : Testosterone, D: Prolactin). Graphs depict data from individual patients with columns indicating median levels $(P$ values after Mann-Whitney test). 


\section{Number of cycles performed}

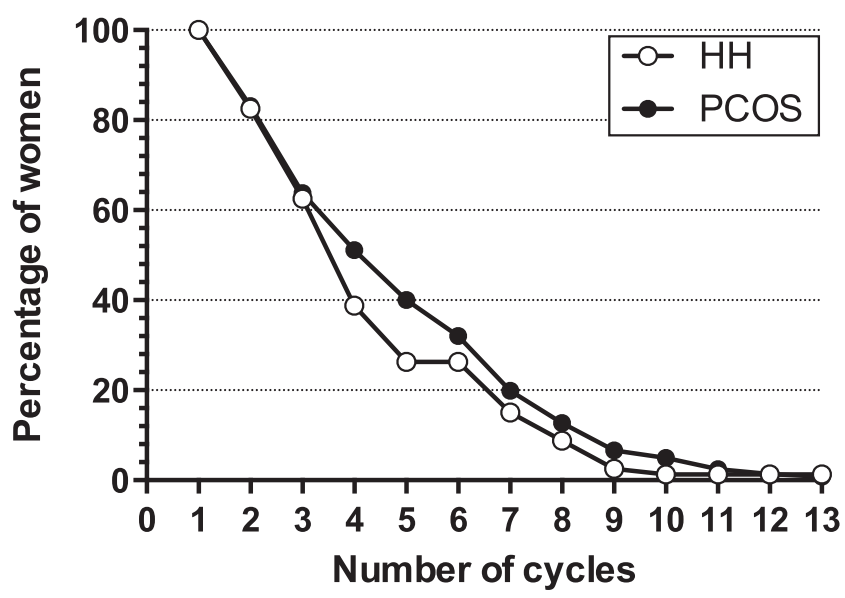

Figure 2 Numbers of cycles performed in women with PCOS and $\mathrm{HH}$. Data show proportion of subjects who received between 1 and 13 cycles.

(maximum daily) dose of FSH or hMG required to stimulate adequate follicle development was strikingly different between PCOS and $\mathrm{HH}$ women (Fig. 3). The threshold dose in the majority of women with PCOS was between 37.5 and $75 \mathrm{IU} /$ day (median: $75 \mathrm{IU} /$ day) whereas in those with $\mathrm{HH}$, most needed more than $75 \mathrm{IU} /$ day (median: $113 \mathrm{IU} /$ day) and a significant proportion of those required doses in excess of $150 \mathrm{IU} /$ day $(19 \% \mathrm{HH}$ vs $7 \%$ PCOS; $P<0.02$, Fisher's exact test) (Fig. 3 ).

The proportion of ovulatory cycles was high in both groups ( $83 \%$ in PCOS, $84 \%$ in $\mathrm{HH}$ ) (Fig. 4A) but, after excluding the small proportion of cycles with uncertain outcome, the percentage of unifollicular ovulatory cycles (defined as a cycle in which the largest subsidiary follicle was $<14 \mathrm{~mm}$ in diameter) was higher in women with PCOS $(77 \%$ vs $53 \%$, Fisher's exact test, $P=0.019$ ) (Fig. 4B). Reciprocally, the risk of multiple follicle development was higher in women with $\mathrm{HH}$. In tandem with the proportion of cycles with multiple
A

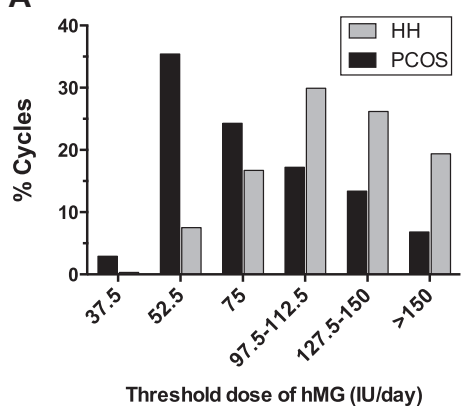

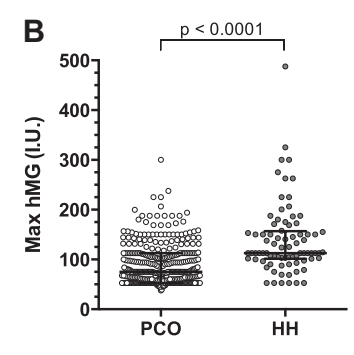

Figure 3 (A) Proportion of cycles in women with either $\mathrm{HH}$ or PCOS according to threshold (maximum daily) dose of gonadotropins. (B) Individual threshold doses showing significantly greater (MannWhitney) requirements of gonadotropin to induce ovulation in $\mathrm{HH}$ than PCOS (bars show median and interquartile range (IQR)). follicle development, the percentage of cycles in which gonadotropin therapy was halted (abandoned cycles) was higher in women with $\mathrm{HH}(25 \%$ vs $15 \%$; Fig. $4 \mathrm{C})$ but in both groups, the main reason for the abandoned cycle was multiple follicle development (Fig. 4D). The length of the 'follicular phase' (i.e. from the start of FSH or hMG treatment to the administration of hCG) was significantly greater, cycle by cycle (Fig. 4E) and overall (Fig. 4E and F) in women with $\mathrm{HH}$.

Serial pelvic ultrasound scans, rather than oestradiol measurements, were the primary basis for assessment of the response to gonadotropins but although we had limited data on changes in oestradiol, endometrial thickness was measured at each visit. We compared endometrial thickness on day of hCG between ovulatory (pregnant and non-pregnant) and anovulatory cycles within groups. In neither group was there a significant difference between non-ovulatory, ovulatory nonpregnant and ovulatory pregnant cycles on day of hCG. However, in cycles that resulted in pregnancy, endometrial thickness in women with $\mathrm{HH}$ was slightly but significantly less than that in subjects with PCOS (median (IQR): HH 7.3 (6.3-8.9); PCOS 9.2 (5.4-11.0) $\mathrm{mm} ; P=0.02$, Mann-Whitney).

We examined the impact of $\mathrm{BMI}$, age and baseline measurements of $\mathrm{LH}$ and testosterone on the threshold dose of $\mathrm{FSH}$ or hMG required to induce ovulation. In women with PCOS, neither age nor BMI had a significant effect on threshold dose (although only five women had a BMI in excess of $30 \mathrm{~kg} / \mathrm{m}^{2}$ ) but those women who had elevated concentrations of either $\mathrm{LH}$ or testosterone required higher doses of FSH/hMG (Fig. 5A, $\mathrm{C}$ and $\mathrm{E})$. In women with $\mathrm{HH}, \mathrm{BMI}$ had no influence on the threshold dose of gonadotropin but women aged 40 years or above required higher doses of gonadotropin as did those whose baseline serum LH level was below the normal range (i.e. $<3.0 \mu / \mathrm{L}$ ) (Fig. 5B, D and F). We found no significant difference between the women with a primary pituitary cause for gonadotropin deficiency and the rest of $\mathrm{HH}$ group in baseline $\mathrm{LH}$, threshold dose of $\mathrm{hMG}$ or length of the follicular phase during the first cycle of treatment.

In women with $\mathrm{HH}$ the length of the follicular phase in the first cycle of treatment was inversely correlated with the pre-treatment $\mathrm{LH}$ and FSH (but not age), whereas there was no such correlation in PCOS subjects (Fig. 6). We had only three cases of severe OHSS (all in women with PCOS).

Forty-nine percent of women with PCOS and $65 \%$ of those with $\mathrm{HH}$ had a least one pregnancy $(P=0.0096$, Fisher's exact test). The cumulative conception data for all cycles are illustrated in Fig. 7 and include further pregnancies in those that had previously conceived. More than $90 \%$ of subjects within each group conceived within six cycles of treatment. Because patients were recruited from around the UK, not all pregnancies were followed to term, so we do not have complete data on 
A

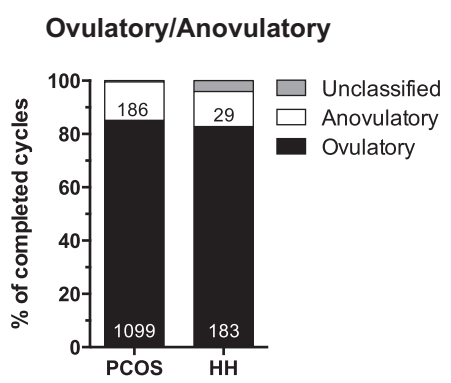

C Completed/Abandoned cycles

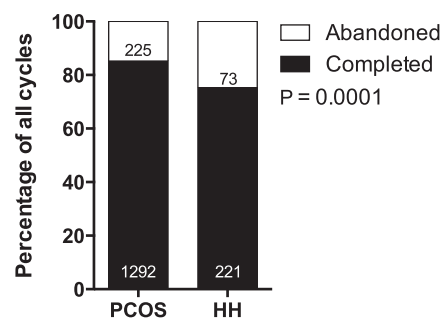

E

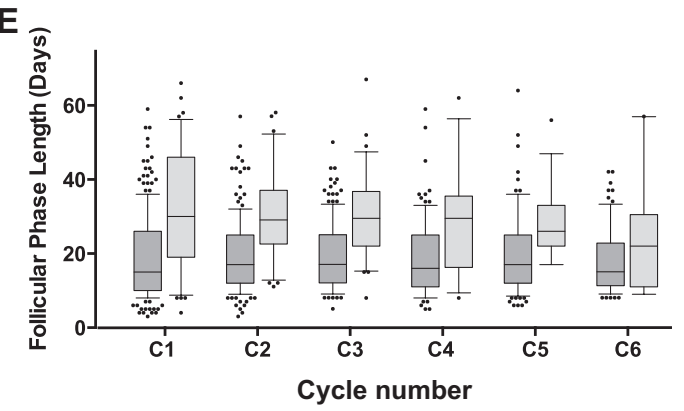

B

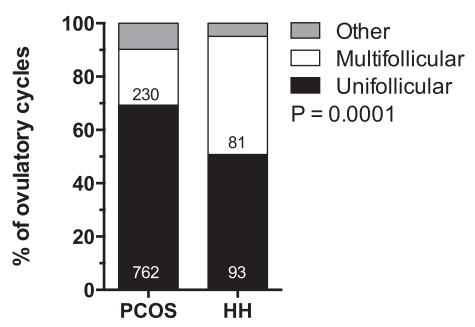

D Abandoned due to multiple or no follicles

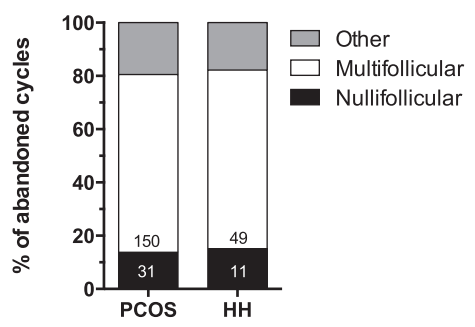

$\mathbf{F}$

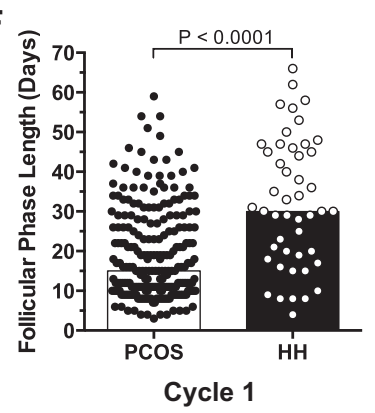

Figure 4 (A) Proportion of cycles that were ovulatory, anovulatory or unclassified (uncertain outcome) in PCOS and $\mathrm{HH}$. (B) Proportion of cycles that were characterised by ovulation of a single follicle, were multi-follicular or showed inadequate follicle development (other). Multi-follicle development was more prevalent in $\mathrm{HH}$ than in PCOS women (Fisher's exact). (C) Abandoned cycles were more common in women with $\mathrm{HH}$ (Fisher's exact) although (D) the percentage in which treatment was stopped because of multiple follicle development (or because no follicle $>10 \mathrm{~mm}$ in diameter developed) was not different between groups. (E) Follicular phase length, cycle by cycle was longer in women with $\mathrm{HH}$ (box and whisker plots denote median, IQR and (95\% confidence intervals) and ( $\mathrm{F}$ ) as was the result when all cycles were taken together (median and individual values; MannWhitney). live birth rate or miscarriage/abortion rate. There are, however, comprehensive data for the first 12 weeks of pregnancy, which allowed us to obtain data on the rates of biochemical and clinical pregnancy rate (i.e. presence of a beating foetal heart on ultrasound) and on the prevalence of multiple pregnancy. The most obvious differences between $\mathrm{HH}$ and PCOS are the higher rates of pregnancy in the former $(78 \%$ vs $44 \% ; P<0.001$, Fisher's exact test). There was, however, a relatively lower incidence of clinical (ongoing) pregnancies in $\mathrm{HH}(66 \%)$, whereas the difference between biochemical and clinical pregnancies in PCOS women was only $4 \%$. The majority of pregnancies occurred within the first three cycles of hMG treatment, and there were very few pregnancies beyond cycle six. Importantly, the rate of multiple pregnancies in each group was small $(0.01 \%$ of cycles and $4 \%$ of pregnancies in PCOS; $0.04 \%$ of cycles and $5 \%$ of pregnancies in $\mathrm{HH}$ (all twins)).

Some informative individual case studies are illustrated in Fig. 8. Two of the women (one with PCOS and one with $\mathrm{HH}$ ) who responded rapidly to low doses of hMG, ovulated a single follicle within 16 days of treatment and became pregnant are shown in Fig. 8A and B. By contrast, two further subjects ovulated one follicle and conceived but required a threshold dose of $150 \mathrm{IU} /$ day or more and had a correspondingly long follicular phase (35 and 48 days, respectively) (Fig. 8C and D). Some women, however, whether PCOS or $\mathrm{HH}$, developed multiple follicles despite a long and gradual 'step-up' dose schedule, and those cycles were abandoned (Fig. 8E and F).

\section{Discussion}

Induction of ovulation using exogenous gonadotropins was introduced more than 50 years ago and, since then, has been applied both to women with PCOS and those with HH (Gemzell et al. 1958, Crooke et al. 1963, 1967, Diczfalusy et al. 1964, Gemzell 1966, Lunenfeld \& Insler 1974). The early use of pituitary FSH was abandoned because of safety issues (in particular, the risk of prion borne disease) but urinary derived preparations soon became available, followed more recently by recombinant human gonadotropins. For the last 20 years, it has become clear that a low-dose, step-up regimen is an effective and safe alternative to the higher dosage schedules typically used until the mid-1990s (Homburg et al. 1995, White et al. 1996, van 
A
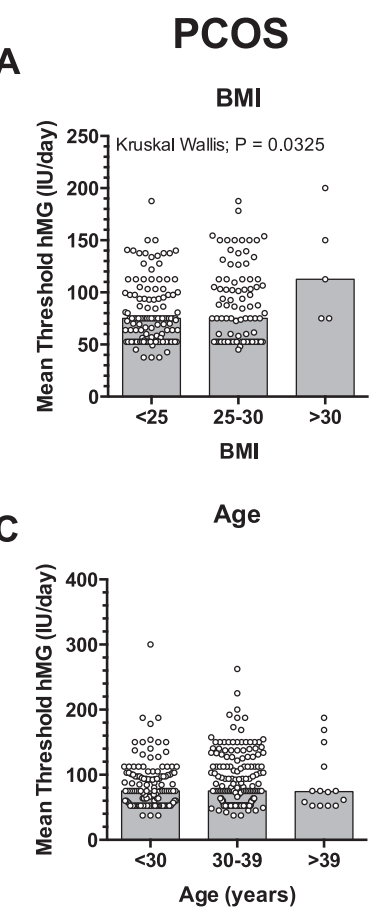

E

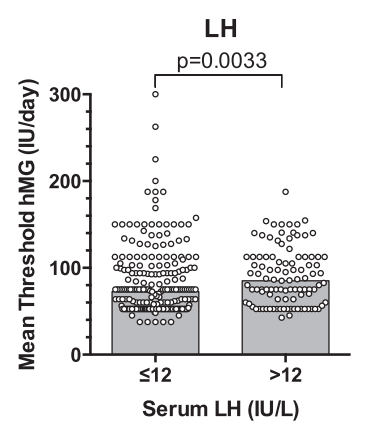

G

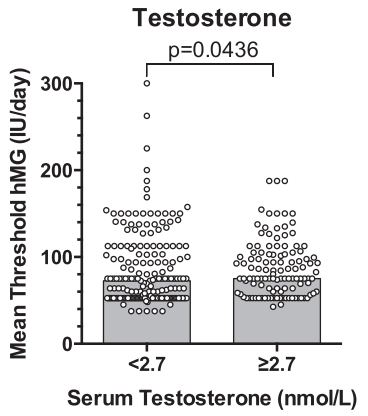

B

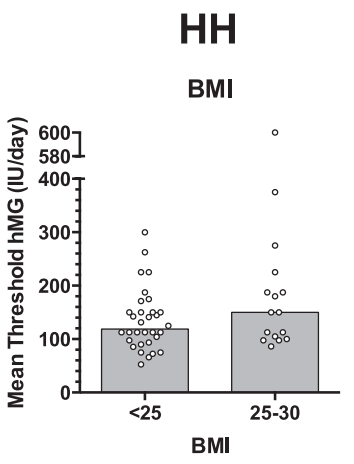

D

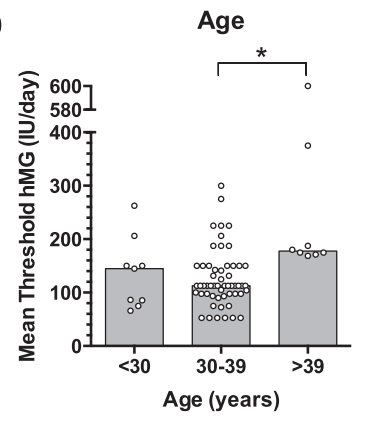

$\mathbf{F}$

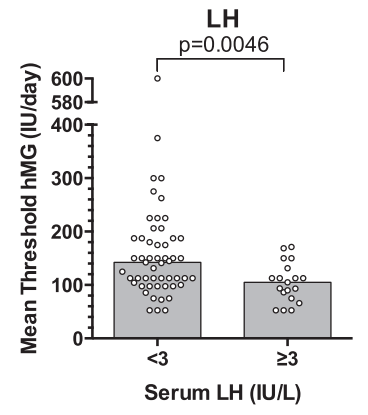

H

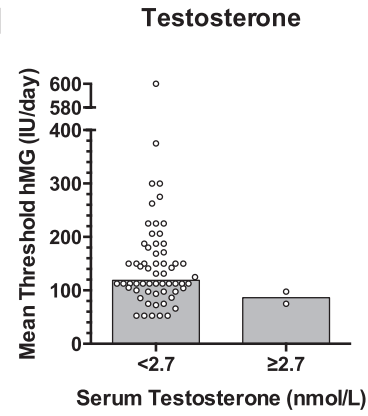

Figure 5 Effect of $B M I(A$ and $B)$, age $(C$ and $D$, baseline $\mathrm{LH}$ (E and $\mathrm{F}$ ) and testosterone $(\mathrm{G}$ and $\mathrm{H})$ on the average threshold dose of gonadotropin in women with PCOS and $\mathrm{HH}$. PCOS women with $\mathrm{BMI}>30 \mathrm{~kg} / \mathrm{m}^{2}$ and women with $\mathrm{LH}$ or testosterone levels that were higher than the laboratory normal range required larger doses of hMG or FSH. Older women with HH (D) and those with lowest baseline LH (F) also needed a higher threshold dose of hMG to induce ovulation.

Santbrink \& Fauser 2006, Li et al. 2010, Yasmin et al. 2013, Balen et al. 2016). Li and colleagues assessed the results of low-dose gonadotropin treatment in 140

women (514 cycles) with PCOS and found similar results with respect to mean threshold dose, duration of treatment, ovulation, pregnancy and multiple pregnancy rates (Li et al. 2010). This group was able to follow pregnancies to term, reporting a live birth rate per cycle of $19.2 \%$. As in our study, the multiple pregnancy rate was low (3\% of clinical pregnancies, all twins), and there was a low incidence of OHSS. However, whereas the low-dose schedule is now well established for management of anovulation in PCOS, there are fewer data for treatment of women with $\mathrm{HH}-$ although a similarly sized group was reported by Balen and colleagues (Balen et al. 1994) - and no extensive study comparing the results in women with PCOS and those with $\mathrm{HH}$. The largest series comparing PCOS and $\mathrm{HH}$ that we could identify was that of Tadokoro and colleagues and that was using conventional doses of gonadotropin (Tadokoro et al. 1997). Furthermore, few studies have looked in detail at clinical and endocrine factors that have an impact on outcome of treatment.

In reviewing this large series of women with anovulatory infertility, we have been able to demonstrate that gonadotropin induction of ovulation, using a lowdose 'step-up' regimen is both effective and safe in both women with PCOS and those with $\mathrm{HH}$. The maximum daily (threshold) dose of FSH or hMG was lower and less variable in women with PCOS than in those with $\mathrm{HH}$ who were a more heterogeneous group, needing overall higher doses and with a longer follicular phase. The dose of hMG and, in parallel, the duration of the follicular phase was inversely related to baseline gonadotropin levels, supporting the view that, as might be predicted, the more gonadotropin-deficient women required a longer period of exposure to exogenous gonadotropins to induce antral follicle growth and maturation. The length of the follicular phase was longest in the first cycle of treatment in the $\mathrm{HH}$ group which is consistent with low baseline gonadotropin levels and therefore, probably, fewer antral follicles from which to recruit. Hypogonadotropic women who required higher gonadotropin doses (>150IU/day) were a little older and had a slightly greater BMI (but were not overweight) than those needing <150IU/day. As we have previously shown (White et al. 1996), women with PCOS who had elevated serum levels of $\mathrm{LH}$ or testosterone required a higher threshold dose of FSH or hMG to induce ovulation. The proportion of overweight and obese women in this series was small when compared to that of $\mathrm{Li}$ and coworkers who found that BMI had little effect on the outcome of treatment in terms of ovulation and pregnancy rates. In our series, obese women with PCOS needed a higher threshold dose of FSH but had similar rates of ovulation and pregnancy to those who were lean or overweight.

A striking feature of the effects of gonadotropin treatment in women with $\mathrm{HH}$ was the higher rate of multiple follicle development than that we observed in 
LH
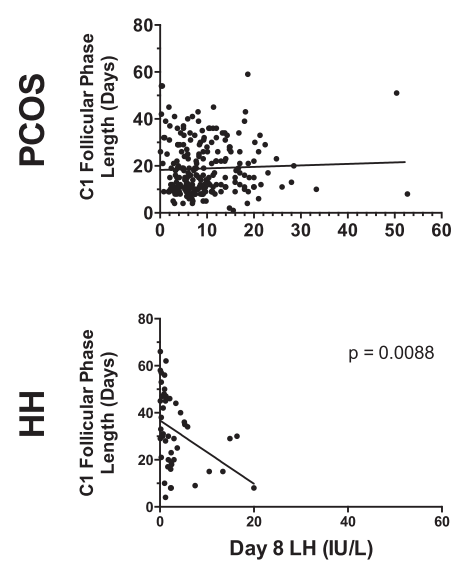

FSH
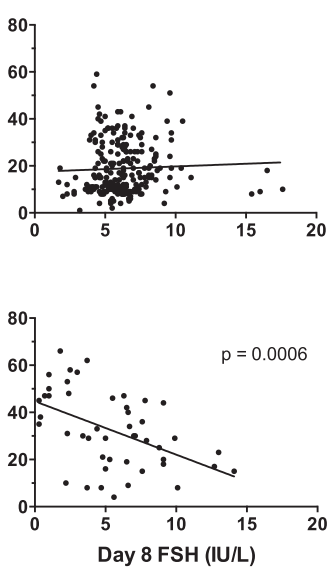

Age

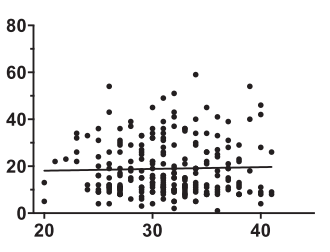

Figure 6 Correlation of length of follicular phase in ovulatory cycles with baseline serum $\mathrm{LH}, \mathrm{FSH}$ concentrations and age in women with $\mathrm{PCOS}$ and $\mathrm{HH}$. Follicular phase length was inversely related to baseline $\mathrm{LH}$ and $\mathrm{FSH}$ in $\mathrm{HH}$ but was unaffected by these factors in PCOS ( $P$ values indicate results of Pearson correlation coefficient). women with PCOS. This is almost certainly related to both the much more variable threshold dose, and the need for higher doses, in $\mathrm{HH}$ cycles. Conversely, the higher rate of unifollicular cycles in PCOS is a function of the lower and narrower range of threshold dose. It was an important policy of the programme to stop gonadotropin treatment if more than 3 large antral follicles developed although, interestingly, the proportion of cycles that was abandoned for this reason was similar in $\mathrm{HH}$ women to that in PCOS subjects. Around $10 \%$ of cycles in both groups were interrupted because of lack of antral follicle growth.

The higher cumulative conception rate in women with $\mathrm{HH}$ is likely to be due largely to the fact that these women had received their first definitive treatment to induce ovulation whereas those with PCOS had, by definition, had previously received clomiphene and either had not ovulated or had not conceived despite ovulating for up to six cycles. In the latter group, it is likely that there were confounding factors contributing to reduced fertility. Sixteen percent of women with PCOS had partners with oligozoospermia and/or had markedly reduced sperm motility, but, interestingly, sperm parameters did not predict outcome of treatment.

One of the most important features of treatment in both groups was the low rate of multiple pregnancies ( $4 \%$ in PCOS, $5 \%$ in $\mathrm{HH}$ ), which is similar to that observed in response to the antioestrogen clomiphene or the aromatase inhibitor letrozole (Legro et al. 2014). All multiples were twins, and there were no higher order pregnancies. These results are similar (using a similar low-dose regimen) to those observed by Li and coworkers (Li et al. 2010) but are in stark contrast to those of older studies in which, then more conventional, higher dose gonadotropin regimens were used (Balen et al. 1994, Tadokoro et al. 1997). Balen and colleagues reported an $18 \%$ prevalence of multiple pregnancies in PCOS although the rate was only $4 \%$ in a population of women with $\mathrm{HH}$ and was similar to that reported here (Balen et al. 1994). By contrast, in the large series reported by Takodoro and co-authors, multiple pregnancies were common in both PCOS women $(20 \%)$ and those with $\mathrm{HH}(30 \%)$ (Tadokoro et al. 1997). An equally important outcome of our series was the very low rate of severe OHSS (only three cases, all PCOS).

Although, in this study, we did not compare the results of gonadotropin treatment with those in response to $\mathrm{GnRH}$, the rates of ovulation, pregnancy and multiple pregnancy as a result of gonadotropin treatment are similar to those reported in large series of women with $\mathrm{HH}$ treated with pulsatile GnRH (Homburg et al. 1989, Martin et al. 1990). Martin and colleagues compared their results of treatment with $\mathrm{GnRH}$ and gonadotropins in women with $\mathrm{HH}$ and reported a multiple pregnancy rate of $15 \%$ in the gonadotropin group (including higher order gestations) and $8 \%$ in the GnRH group, albeit using more conventional doses of gonadotropin (Martin et al. 1993). Interestingly, the rate of severe OHSS was low in that series, as it was in that reported by Balen and coworkers, illustrating in accord with our study, that OHSS in women with $\mathrm{HH}$ is uncommon (Balen et al. 1994).

Because our induction of ovulation clinic accepted referrals from all over the United Kingdom (as well as providing a local service), we have incomplete data on

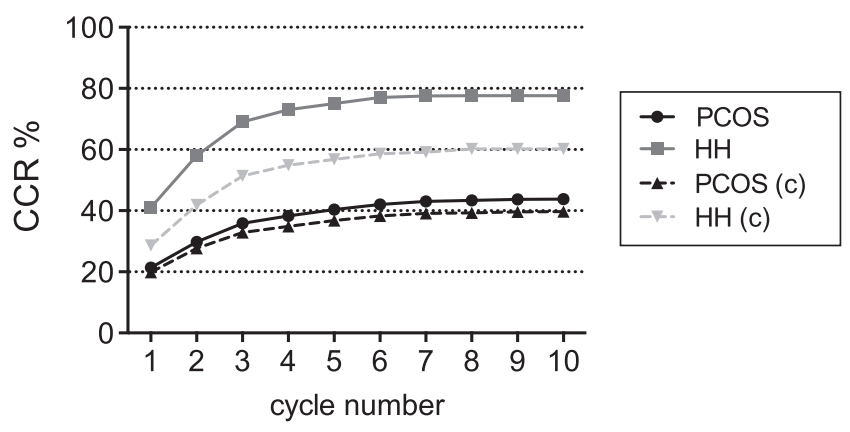

Figure 7 Cumulative conception rate in women with $\mathrm{HH}$ and PCOS. Solid lines indicate pregnancy rate as detected by serum or urine hCG and broken lines show clinical pregnancy rate (c). 
PCOS

A

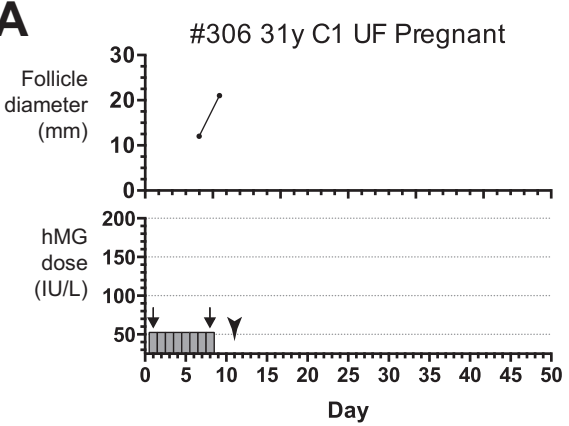

C
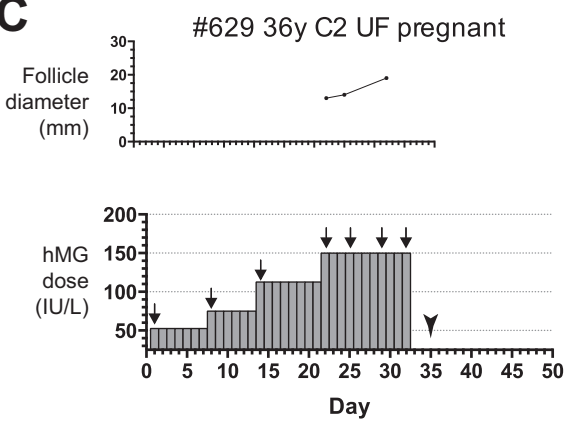

E

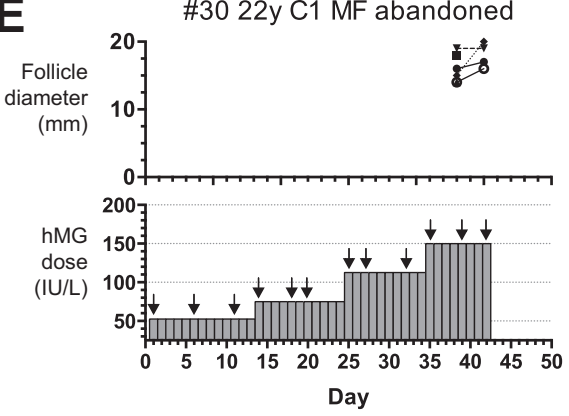

$\mathrm{HH}$

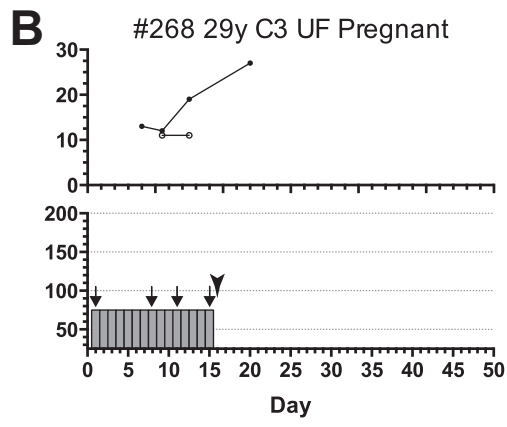

D

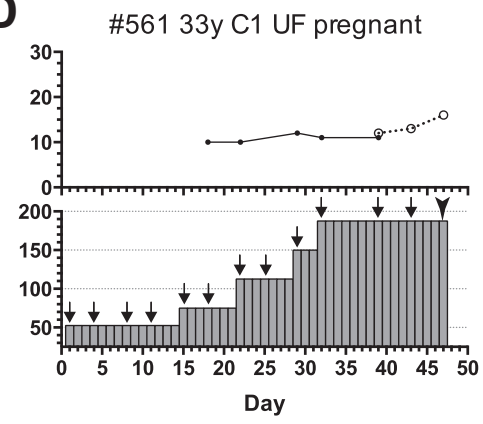

$\mathbf{F}$

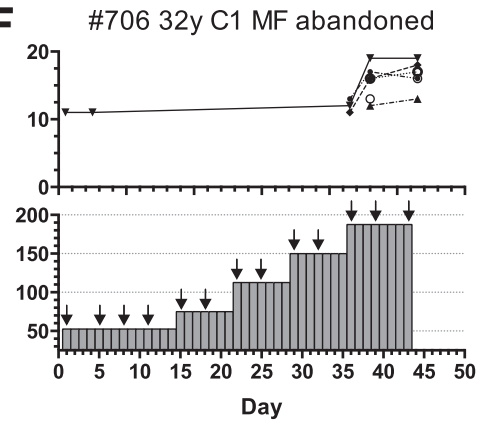

Figure 8 Individual illustrations of various responses to treatment in 3 women with PCOS and 3 with $\mathrm{HH}$. Patients \#306 and \#268 (A and $\mathrm{B}$ ) had a mono-follicular, ovulatory cycle resulting in pregnancy with a threshold hMG dose of $50 \mathrm{IU} /$ day and $75 \mathrm{IU} /$ day respectively. Patients \#629 and \#561 (C and D) similarly became pregnant after mono-follicular ovulation but needed higher doses of hMG (150 IU/day and $175 \mathrm{IU} /$ day, respectively). By contrast patients \#\#30 and \#706 (E and F) developed multiple follicles despite a slow, step-up increment in hMG dose and the cycle was abandoned in each case. Shaded bars indicate daily dose of hMG; arrows with tails indicate days of ultrasound scan; arrowhead shows day of ovulatory hCG dose; closed circles connected with lines show progression of dominant follicle development; open circles and line in panel $\mathrm{B}$ indicate a subsidiary follicle that did not increase in size; other symbols and lines in figs $E$ and $F$ show track of multiple follicles. pregnancy outcome, but we are able to report more comprehensive information about early pregnancy loss. In particular, we were able to provide data on the differences between those cycles that were characterised by a positive (hCG) pregnancy test and those that resulted in an ongoing, clinical pregnancy (beating foetal heart). In women with PCOS, the difference between biochemical and clinical pregnancies was small. By contrast, in women with $\mathrm{HH}$, although the clinical pregnancy rate was high, the biochemical pregnancy rate was strikingly higher. We considered three possible explanations for this disparity: (1) that $\mathrm{HH}$ pregnancies were more prone to failure of implantation, perhaps due to a sub-optimal quality of either embryos or of endometrial environment, (2) that cycles in $\mathrm{HH}$ women were subject to more intensive post-ovulation monitoring, resulting in a high early detection rate or (3) that interpretation of a positive hCG test was compromised by the fact that $\mathrm{HH}$ women received luteal support in the form of further hCG injections. The latter is perhaps the most plausible explanation because even though the pregnancy test was performed 5-8 days after the hCG ovulation trigger, the long half-life in the circulation (Chan et al. 2003) means that any residual hCG may have given a false-positive result. The second possible reason (closer monitoring) was not sustainable; monitoring post-ovulation was similar in both groups. We cannot, however, rule out the possibility of more abnormalities in embryos or endometrial environment. Our previous publication reporting an earlier analysis of results in women with PCOS suggests that the rate of pregnancy loss following low-dose gonadotropin treatment $(23 \%)$ is no greater than in the general population (Gorry et al. 2006). This is supported by data from a population study, which showed no significant difference between women with symptoms of PCOS and the reference population in the rate of spontaneous abortions (24\% vs 20\%) (Koivunen et al. 2008). 
It is clear from these findings that the primary choice for induction of ovulation in clomiphene-resistant women PCOS should be low-dose gonadotropin treatment as recommended by the ESHRE/ASRM Consensus Workshop Group (Thessaloniki 2008) and not, as has become all too familiar in clinical practice, IVF. Similarly, low-dose gonadotropin therapy (as an alternative to pulsatile $\mathrm{GnRH}$ ) is a safe and effective means of restoring fertility in women with $\mathrm{HH}$. Obviously, IVF may be indicated in those couples in whom there is an additional cause of infertility such as tubal disease or male factor infertility but should be discouraged as the default mode of treatment when restoration of ovulation is the more physiological (and clearly effective) method.

\section{Declaration of interest}

The authors declare that there is no conflict of interest that could be perceived as prejudicing the impartiality of this review.

\section{Funding}

This work was performed with the aid of Infrastructure Support Funding from the National Institute for Health Research (NIHR) Imperial Biomedical Research Centre (BRC).

\section{Author contribution statement}

Davinia White was the lead clinician in charge of the induction of ovulation service and was responsible for recording patient data and creating the initial electronic database from which these results were derived. Susannah Lovelock was a medical student undertaking her intercalated BSc project who checked the patient records, reorganised and transferred the cleaned data to FileMaker Pro. Kate Hardy created a customised data base in FileMaker Pro and was primarily responsible for refinement, constraint, stratification and sub-analysis of the data. She also produced figures for the paper and contributed to the writing of the manuscript. Stephen Franks set up the induction of ovulation clinic and was involved in its management throughout. He contributed to the analysis of data and organisation of the results for publication. He was primarily responsible for writing the manuscript.

\section{Acknowledgements}

The authors would like to thank David Polson, Debbie Kiddy, Hazel Watson, Peggy Sagle, Diana Hamilton-Fairley, Carole Gilling-Smith, Eleni Kousta and Lisa Webber for their invaluable contributions to running the Induction of Ovulation service at St Mary's over many years.

\section{References}

Balen AH, Braat DD, West C, Patel A \& Jacobs HS 1994 Cumulative conception and live birth rates after the treatment of anovulatory infertility: safety and efficacy of ovulation induction in 200 patients. Human Reproduction 9 1563-1570. (https://doi.org/10.1093/ oxfordjournals.humrep.a138750)
Balen AH, Morley LC, Misso M, Franks S, Legro RS, Wijeyaratne CN, Stener-Victorin E, Fauser BC, Norman RJ \& Teede H 2016 The management of anovulatory infertility in women with polycystic ovary syndrome: an analysis of the evidence to support the development of global WHO guidance. Human Reproduction Update 22 687-708. (https://doi.org/10.1093/humupd/dmw025)

Brown JB, Catt KJ \& Martin FI 1967 Preparation of gonadotrophin and growth hormone from human pituitaries for clinical use. Journal of Endocrinology 38 451-454. (https://doi.org/10.1677/ joe.0.0380451)

Chan CC, Ng EH, Chan MM, Tang OS, Lau EY, Yeung WS \& Ho PC 2003 Bioavailability of hCG after intramuscular or subcutaneous injection in obese and non-obese women. Human Reproduction 18 2294-2297. (https://doi.org/10.1093/humrep/deg446)

Crooke AC 1970 Induction of ovulation with gonadotrophins. British Medical Bulletin 26 17-21. (https://doi.org/10.1093/oxfordjournals.bmb. a070736)

Crooke AC, Butt WR, Palmer RF, Morris R, Edwards RL \& Anson CJ 1963 Clinical trial of human gonadotrophins. I. The effect of pituitary and urinary follicle stimulating hormone and chorionic gonadotrophin on patients with idiopathic secondary amenorrhoea. Journal of Obstetrics and Gynaecology of the British Commonwealth 70 604-635. (https:// doi.org/10.1111/j.1471-0528.1963.tb04957.x)

Crooke AC, Butt WR, Bertrand PV \& Morris R 1967 Treatment of infertility and secondary amenorrhoea with follicle-stimulating hormone and chorionic gonadotrophin. Lancet 2 636-637. (https://doi.org/10.1016/ S0140-6736(67)90683-6)

Diczfalusy E, Johannisson E, Tillinger KG \& Bettendorf G 1964 Comparison of the clinical and steroid metabolic effect of human pituitary- and urinary gonadotrophins in amenorrhoeic women. Acta Endocrinologica 45 (Supplement 90) 35-56.

Franks S \& Gilling-Smith C 1994 Advances in induction of ovulation. Current Opinion in Obstetrics and Gynecology 6 136-140.

Gemzell C 1966 Human pituitary gonadotropins in the treatment of sterility. Fertility and Sterility 17 149-159. (https://doi.org/10.1016/ S0015-0282(16)35880-0)

Gemzell CA, Diczfalusy E \& Tillinger G 1958 Clinical effect of human pituitary follicle-stimulating hormone (FSH). Journal of Clinical Endocrinology and Metabolism 18 1333-1348. (https://doi.org/10.1210/ jcem-18-12-1333)

Gorry A, White DM \& Franks S 2006 Infertility in polycystic ovary syndrome: focus on low-dose gonadotropin treatment. Endocrine $\mathbf{3 0}$ 27-33. (https://doi.org/10.1385/ENDO:30:1:27)

Homburg R, Eshel A, Armar NA, Tucker M, Mason PW, Adams J, Kilborn J, Sutherland IA \& Jacobs HS 1989 One hundred pregnancies after treatment with pulsatile luteinising hormone releasing hormone to induce ovulation. BMJ 298 809-812. (https://doi.org/10.1136/ bmj.298.6676.809)

Homburg R, Levy T \& Ben-Rafael Z 1995 A comparative prospective study of conventional regimen with chronic low-dose administration of folliclestimulating hormone for anovulation associated with polycystic ovary syndrome. Fertility and Sterility 63 729-733. (https://doi.org/10.1016/ S0015-0282(16)57473-1)

Kamrava MM, Seibel MM, Berger MJ, Thompson I \& Taymor ML 1982 Reversal of persistent anovulation in polycystic ovarian disease by administration of chronic low-dose follicle-stimulating hormone. Fertility and Sterility 37 520-523. (https://doi.org/10.1016/S00150282(16)46159-5)

Koivunen R, Pouta A, Franks S, Martikainen H, Sovio U, Hartikainen AL, McCarthy MI, Ruokonen A, Bloigu A, Jarvelin MR et al. 2008 Fecundability and spontaneous abortions in women with self-reported oligo-amenorrhea and/or hirsutism: Northern Finland Birth Cohort 1966 study. Human Reproduction 23 2134-2139. (https://doi.org/10.1093/ humrep/den136)

Kousta E, White DM \& Franks S 1997 Modern use of clomiphene citrate in induction of ovulation. Human Reproduction Update 3 359-365. (https://doi.org/10.1093/humupd/3.4.359)

Legro RS, Brzyski RG, Diamond MP, Coutifaris C, Schlaff WD, Casson P, Christman GM, Huang H, Yan Q, Alvero R et al. 2014 Letrozole versus clomiphene for infertility in the polycystic ovary syndrome. New England Journal of Medicine 371 119-129. (https://doi.org/10.1056/ NEJMoa1313517) 
Li HW, Van Esch M, De Vries J, Duncan WC \& Anderson RA 2010 Gonadotrophin ovulation induction is a successful treatment for World Health Organisation Group II anovulatory subfertility in women aged up to 40 and with body mass index up to 34. Human Fertility 13 35-40. (https://doi.org/10.3109/14647270903490765)

Lunenfeld B \& Insler V 1974 Classification of amenorrhoeic states and their treatment by ovulation induction. Clinical Endocrinology 3 223-237. (https://doi.org/10.1111/j.1365-2265.1974.tb01799.x)

Martin K, Santoro N, Hall J, Filicori M, Wierman M \& Crowley WF Jr 1990 Clinical review 15: management of ovulatory disorders with pulsatile gonadotropin-releasing hormone. Journal of Clinical Endocrinology and Metabolism 71 1081A-1081G. (https://doi.org/10.1210/jcem-71-51081)

Martin KA, Hall JE, Adams JM \& Crowley WF Jr 1993 Comparison of exogenous gonadotropins and pulsatile gonadotropin-releasing hormone for induction of ovulation in hypogonadotropic amenorrhea. Journal of Clinical Endocrinology and Metabolism 77 125-129.

Polson DW, Mason HD, Saldahna MB \& Franks S 1987 Ovulation of a single dominant follicle during treatment with low-dose pulsatile follicle stimulating hormone in women with polycystic ovary syndrome. Clinical Endocrinology 26 205-212. (https://doi.org/10.1111/j.1365-2265.1987. tb00778.x)

Rotterdam ESHRE/ASRM-Sponsored PCOS Consensus Workshop Group 2004. Revised 2003 consensus on diagnostic criteria and long-term health risks related to polycystic ovary syndrome. Fertility and Sterility 81 19-25. (https://doi.org/10.1016/j.fertnstert.2003.10.004)

Schwartz M, Jewelewicz R, Dyrenfurth I, Tropper P \& Vande Wiele RL 1980 The use of human menopausal and chorionic gonadotropins for induction of ovulation. Sixteen years' experience at the Sloane Hospital for Women. American Journal of Obstetrics and Gynecology 138 801-807. (https://doi.org/10.1016/S0002-9378(16)32740-5)

Tadokoro N, Vollenhoven B, Clark S, Baker G, Kovacs G, Burger H \& Healy D 1997 Cumulative pregnancy rates in couples with anovulatory infertility compared with unexplained infertility in an ovulation induction programme. Human Reproduction 12 1939-1944. (https:// doi.org/10.1093/humrep/12.9.1939)

Thessaloniki EA-SPCWG 2008 Consensus on infertility treatment related to polycystic ovary syndrome. Human Reproduction 23 462-477. (https:// doi.org/10.1093/humrep/dem426)

van Santbrink EJ \& Fauser BC 2006 Ovulation induction in normogonadotropic anovulation (PCOS). Best Practice and Research: Clinical Endocrinology and Metabolism 20 261-270. (https://doi. org/10.1016/j.beem.2006.03.002)

White DM, Polson DW, Kiddy D, Sagle P, Watson H, Gilling-Smith C, Hamilton-Fairley D \& Franks S 1996 Induction of ovulation with lowdose gonadotropins in polycystic ovary syndrome: an analysis of 109 pregnancies in 225 women. Journal of Clinical Endocrinology and Metabolism 81 3821-3824.

Yasmin E, Davies M, Conway G, Balen AH \& British Fertility S 2013 British Fertility Society. 'Ovulation induction in WHO Type 1 anovulation: guidelines for practice'. Produced on behalf of the BFS Policy and Practice Committee. Human Fertility 16 228-234. (https://doi.org/10.3 109/14647273.2013.829673)

Zwart-van Rijkom JE, Broekmans FJ \& Leufkens HG 2002 From HMG through purified urinary $\mathrm{FSH}$ preparations to recombinant $\mathrm{FSH}$ : a substitution study. Human Reproduction 17 857-865. (https://doi. org/10.1093/humrep/17.4.857)

Received 14 November 2017

First decision 2 December 2017

Revised manuscript received 9 February 2018

Accepted 19 February 2018 